\title{
Pattern of antibiotic resistant mastitis in dairy cows
}

\author{
D. Chandrasekaran, P. Venkatesan, K. G. Tirumurugaan, A. P. Nambi, P. S. Thirunavukkarasu, K. Kumanan,
} S. Vairamuthu and S. Ramesh

Department of Veterinary Clinical Medicine, Ethics and Jurisprudence,

Madras Veterinary College, Tamil Nadu Veterinary and Animal Sciences University (TANUVAS), Tamil Nadu, India.

Corresponding author: D. Chandrasekaran, email:drchandrus@yahoo.com

PV: venkatesanperiasamy@gmail.com, KGT: tirumurugaankg@tanuvas.org.in, APN: nambi529@yahoo.com, PST:

pstthiru@yahoo.com, KK: kumananrani@hotmail.com, SV: vairamuthu@tanuvas.org.in, SR: ramesh@tanuvas.org.in

Received: 24-03-2014, Revised: 08-05-2014, Accepted: 11-05-2014, Published online: 10-06-2014

doi: $10.14202 /$ vetworld.2014.389-394

How to cite this article: Chandrasekaran D, Venkatesan P, Tirumurugaan KG, Nambi AP, Thirunavukkarasu PS, Kumanan K, Vairamuthu S and Ramesh S (2014) Pattern of antibiotic resistant mastitis in dairy cows, Veterinary World 7(6): 389-394.

\begin{abstract}
Aim : To study the prevalence of drug resistant mastitis and their pattern of antibiotic resistance in dairy cows from Tamil Nadu.

Materials and Methods: Isolation and identification of resistant pathogens were performed from acute clinical mastitis samples. Based on culture, isolation and sensitivity tests, cows with resistant mastitis were grouped as; Group I: Escherichia $\operatorname{coli}(\mathrm{n}=119)$, Group II: Staphylococcus aureus ( $\mathrm{n}=104)$ and Group III: Methicillin-resistant Staphylococcal aureus (MRSA) $(\mathrm{n}=12)$. The isolates were tested using agar disc diffusion method for their antimicrobial susceptibility and modified resazurin assay microdilution technique for minimum inhibitory concentration (MIC) to 8 antimicrobial drugs. The organisms were also confirmed for their identity by performing PCR on the bacterial pellet targeting the specific genes such as 16s-23s rRNA, $m e c \mathrm{~A}$ and blaZ respectively for the resistant pathogens and also confirmed by sequencing.

Results: Antibiotic resistant mastitis was detected in 235 out of 401 cows accounting to $56.1 \%$. The predominant resistant causative pathogen was E. coli (50.64\%) followed by S. aureus (44.25\%) and MRSA (5.11\%). In vitro antibiotic sensitivity test and MIC breakpoints, E. coli, S. aureus and MRSA organisms showed more sensitivity to enrofloxacin, amoxicillin + sulbactam, gentamicin and ceftriaxone and had highest resistant to penicillin followed by amoxicillin, oxytetracycline and methicillin. E. coli and S. aureus isolates were found to be resistant to 1 or 2 antimicrobials, whereas most of the MRSA isolates were found to be multi-drug resistant i.e resistance to 3 or more of antimicrobials. Out of 235 milk samples, the specific target gene 16s-23s rRNA (E. coli ), 16s-23s rRNA (S. aureus) and MRSA (mecA and blaZ) could be amplified from 119,104 and 12 isolates with a percentage positivity of $50.64(119 / 235), 89.64(104 / 116)$ and $10.34(12 / 116)$ respectively.
\end{abstract}

Conclusion: Prevalence of antimicrobial resistance (AMR) in bovine mastitis pathogens was high. Most MRSA pathogens were multidrug resistant. E. coli and S. aureus isolates were resistant to few antimicrobials.

Keywords: bovine resistant mastitis, Escherichia coli, methicillin resistant Staphylococcus aureus, Staphylococcus aureus.

\section{Introduction}

Mastitis is the most common and economically significant disease affecting dairy cattle. A variety of bacteria can be isolated from bovine mastitis cases. Staphylococcus aureus and Escherichia coli are the most common causes of contagious and environmental clinical mastitis, respectively. Antimicrobial therapy is commonly implemented for mastitis prevention and control. Unfortunately, despite the best possible antimicrobial treatments, failures of bacteriological cure are common, especially for $S$. aureus mastitis and antimicrobial resistance (AMR) which is considered to be one of the reasons for low cure rates [1]. Additionally, AMR in bacteria is a public health hazard, and extensive use of antimicrobial is considered a potentially important driver of AMR. Several strains of $S$. aureus isolated from mastitis case have been reported to show resistance against multiple antimicrobials such as penicillin-G,

Copyright: The authors. This article is an open access article licensed under the terms of the Creative Commons Attribution License (http://creativecommons.org/licenses/by/2.0) which permits unrestricted use, distribution and reproduction in any medium, provided the work is properly cited. gentamicin, streptomycin, ampicillin, ciprofloxacin, oxytetracycline [2].

Beta-lactam antibiotics are frequently used in mastitis therapy and the resistance is due to the production of beta-lactamases and low-affinity penicillin-binding protein, PBP2A [3]. $\beta$-lactamase resistant penicillins such as methicillin and oxacillin are not used in dairy cows except for cloxacillin that is used in products for intramammary administration [4]. Methicillin-resistant S. aureus (MRSA) have been isolated from mastitis milk samples and have the potential to complicate treatment of bovine mastitis [5]. The presence of MRSA in bovine mastitis is a potential risk to other exposed cattle and farm workers including veterinarians [6]. In general, the emergence and transfer of AMR bacteria or genetic determinants from animals to human populations via food chain is a growing concern [7]. Comprehensive information on the prevalence of AMR in bovine mastitis pathogens in milk is lacking in India.

The objective of the present study was to study the prevalence of drug resistant mastitis and their pattern of antibiotic resistance in dairy cows from 
Table-1: primers used in the study

\begin{tabular}{|c|c|c|c|c|}
\hline Primer & Primer sequence & Organism targeted & Amplicon size & Reference \\
\hline $\begin{array}{l}\text { SU-F } \\
\text { SU-R }\end{array}$ & $\begin{array}{l}\text { 5' TTC GTA CCA GCC AGA GGT GGA 3' } \\
5^{\prime} \text { TCT TCA GCG CAT CAC CAA TGC C 3' }\end{array}$ & S. aureus & $229 \mathrm{bp}$ & {$[10]$} \\
\hline Eco 2083 & 5' GCT TGA CAC TGA ACA TTG AG 3' & E. coli & $662 \mathrm{bp}$ & [11] \\
\hline $\begin{array}{l}\text { Eco } 2745 \\
\operatorname{mecA}\end{array}$ & $\begin{array}{l}\text { 5' GCA CTT ATC TCT TCC GCA TT 3' } \\
\text { 5' AAA ATC GAT GGT AAA GGT TGG C 3' } \\
\text { 5'AGT TCT GCA GTA CCG GAT TTG C 3' }\end{array}$ & $\begin{array}{l}\text { Methicillin resistant } \\
\text { S. aureus (MRSA) }\end{array}$ & $533 \mathrm{bp}$ & [12] \\
\hline blaZ & $\begin{array}{l}\text { 5' ACT TCA ACA CCT GCT GCT TTC 3' } \\
\text { 5' TGA CCA CTT TTA TCA GCA ACC 3' }\end{array}$ & $\begin{array}{l}\text { Methicillin resistant } \\
\text { S. aureus (MRSA) }\end{array}$ & $173 \mathrm{bp}$ & [13] \\
\hline
\end{tabular}

Tamil Nadu, India.

\section{Materials and Methods}

Sampling and bacterial culturing: Four hundred and one milk samples were collected from acute mastitis cows from Large Animal Clinic Medicine Unit of Madras Veterinary College Teaching Hospital and six dairy farms in Coimbatore district. The guidelines of National Mastitis Council (NMC) were followed for sample collection, transportation, culture and isolation of bacteria. $S$. aureus isolates were identified as grampositive cocci by Gram stain, growth on blood agar and mannitol salt agar and a positive test for catalase and coagulase [8]. Escherichia coli isolates were identified as gram negative rods by Gram stain, lactose fermenters on MacConkey agar, and a negative oxidase test. Based on incidence of common causative pathogens and sensitivity tests, isolates were categorized as resistant i.e exhibiting in vitro resistance to 1 or 2 antimicrobials and multidrug-resistant i.e exhibiting in vitro resistance to 3 or more antimicrobials. Cows with resistant mastitis were grouped as; Group I: Escherichia coli $(\mathrm{n}=119)$, Group II: Staphylococcus aureus $(\mathrm{n}=104)$ and Group III: MRSA $(\mathrm{n}=12)$.

Antibiotic susceptibility and resistance: Antimicrobial susceptibility testing was carried out with equivalence of $0.5 \mathrm{McFarland}$ turbidity standard by agar disc diffusion method on Mueller-Hinton agar plates following the guidelines of Clinical and Laboratory Standards Institute (CLSI) [8]. All the bacteria isolated were tested in vitro for their sensitivity to 8 different antibiotics, that are commonly used in veterinary practice. Commercially available antibiotic discs (Himedia, Mumbai) were used in the study viz., enrofloxacin (10 mcg), amoxicillin + sulbacatm (15 $\mathrm{mcg})$, amoxicillin (10 mcg), gentamicin (10 mcg), ceftriaxone $(30 \mathrm{mcg})$, oxytetracycline $(30 \mathrm{mcg})$ and penicillin $\mathrm{G}$ (10 units) and oxacillin ( $5 \mathrm{mcg})$. The sizes of the zone of inhibition were recorded and interpreted as either susceptible or resistant to the exposed agent.

Minimum inhibitory concentration (MIC): The minimum inhibitory concentrations of different antibiotics for $E$. coli, S. aureus and MRSA isolates were determined by modified resazurin assay microdilution technique [9]. The MIC panels consisted of dehydrated antimicrobial agents: amoxicillin, ceftriaxone, enrofloxacin, gentamicin, pencillin, oxytetracycline, amoxicillin + sulbactam and oxacillin in 96-well U bottom microtiter plates and performed in accordance with the guidelines established by the CLSI [8]. The lowest concentration of antibiotic that resulted in complete inhibition of visible growth and did not produce any turbidity was taken as the MIC end point.

Polymerase chain reaction (PCR) for identification of the mastitis causing bacteria: A single colony with typical morphology from the selective agar was suspended in nuclease free water and lysed by boiling for 10 minutes and the lysate was stored at $-20^{\circ} \mathrm{C}$ until use. The lysate were used in a PCR reaction with primers targeting the specific gene for different strains. The PCR amplicons from some of the samples were sequenced and analysed by BLAST search to confirm their identity. The details of primers used in the study are described in Table-1.

PCR amplification was performed in a total reaction volume of $25 \mu \mathrm{l}$. The reaction mixture contained 12.5 $\mu l$ of the master mix, $20 \mathrm{pmol}$ of the forward and reverse primer and $1.5 \mu \mathrm{l}$ of the test lysate. The amplification profile for the detection of different genes were $94^{\circ} \mathrm{C} 5 \mathrm{~min}$; 35 cycles of $95^{\circ} \mathrm{C}$ for $45 \mathrm{sec}$, $55^{\circ} \mathrm{C}$ for $1 \mathrm{~min}, 72^{\circ} \mathrm{C}$ for $1 \mathrm{~min}$; final extension of $72^{\circ} \mathrm{C}$ for $7 \mathrm{~min}$. The PCR products were separated by gel electrophoresis in 1.5 per cent agarose gel using $1 \mathrm{x}$ Tris acetate EDTA buffer along with standard DNA marker (100 bp ladder, Genei, Bangalore) and visualized with ethidium bromide staining.

\section{Results}

Out of 401 clinical mastitis samples subjected to bacterial isolation, $184(45.89 \%)$ were positive for $E$ coli, 162 (40.4\%) were positive for S. aureus, 12 (2.99 $\%)$ were positive for MRSA, 14 (3.49\%) were positive for Bacillus spp., 13 (3.24\%) were positive for Streptococcus spp. and 16 (3.99\%) samples showed mixed infection. Mixed infections were not exhibiting resistant to antibiotics (Pansusceptible).

Antibiotic resistant mastitis was detected in 235 out of 401 cows accounting to $56.1 \%$. The predominant resistant causative pathogen was E. coli $(50.64 \%)$ followed by $S$. aureus (44.25\%) and MRSA (5.11\%).

Antibiotic susceptibility and resistance: $E$. coli showed more sensitivity to enrofloxacin $(79 \%)$ followed by amoxicillin and sulbactam (74\%), gentamicin (73.1\%) and ceftriaxone (69\%). The isolates had highest resistance to penicillin (63\%) followed by amoxicillin (52.1\%), oxytetracycline (47.95) and methicillin (45.4\%). Most of the E.coli isolates (86.55\%) were found to be resistant i.e resistance to 1 or 2 antimicro- 
Table-2: MIC of drugs against $E$. coli $(n=119)$ isolated from udder of cows

\begin{tabular}{|c|c|c|c|c|c|c|c|c|c|c|c|c|}
\hline \multirow[t]{2}{*}{ Antibiotics } & \multicolumn{12}{|c|}{$\operatorname{MIC}(\mu \mathrm{g} / \mathrm{mIL}) \quad(\mathrm{n}=119)$} \\
\hline & 125 & 62.5 & 31.25 & 15.62 & 7.8 & 3.9 & 1.95 & 0.97 & 0.48 & MIC Range & MIC $_{-50}$ & MIC $_{-90}$ \\
\hline Gentamicin & 9.2 & 11 & 2.5 & 3.4 & 11.7 & 5.9 & 16 & 27.7 & 12.6 & $0.48-125$ & 1.95 & 62.5 \\
\hline Oxytetracycline & 17.7 & 25.2 & 15.1 & 5.9 & | 16 & 6.7 & 8.4 & 5 & - & $0.97-125$ & 31.25 & 125 \\
\hline Ceftriaxone & 4.2 & 9.2 & 20.2 & 3.4 & 12.6 & 1.7 & 13.4 & 20.2 & 15.1 & $0.48-125$ & 3.9 & 62.5 \\
\hline Enrofloxacin & 8.4 & 3.4 & 12.6 & 4.2 & 11.7 & 3.4 & | 21.8 & 18.5 & 16 & $0.48-125$ & 1.95 & 62.5 \\
\hline Amoxicillin & 22.7 & 30.3 & 6.7 & 23.5 & 6.7 & 2.5 & 7.6 & - & - & $1.95-125$ & 62.5 & 125 \\
\hline Pencillin G & 19.3 & 41.2 & 8.4 & 12.6 & 5.9 & 4.2 & 7.6 & 0.8 & - & $0.95-125$ & 62.5 & 125 \\
\hline $\begin{array}{l}\text { Amoxicillin + } \\
\text { Sulbactam }\end{array}$ & - & 0.8 & 31.1 & 0.8 & 8.4 & 8.4 & 31.1 & 12.6 & 6.7 & $0.48-125$ & 1.95 & 31.25 \\
\hline Oxacillin & 6.7 & 33.6 & 16 & 17.7 & 5 & 14.3 & 6.7 & - & - & $1.95-125$ & 31.25 & 62.5 \\
\hline
\end{tabular}

Numbers indicate percentage of isolates. Numbers in bold indicate percentage of isolates with MIC values greater than the highest concentration in the dilution range and vertical line indicate clinical breakpoints, with the value to the right of the line being susceptible and those to the left being resistant. MIC $_{50}$ and MIC $_{90}$ values are concentrations at which $\geq 50 \%$ and $\geq 90 \%$ of isolates are inhibited respectively.

Table-3: MIC of drugs against S.aureus $(n=104)$ isolated from udder of cows

\begin{tabular}{|c|c|c|c|c|c|c|c|c|c|c|c|c|}
\hline \multirow[t]{2}{*}{ Antibiotics } & \multicolumn{12}{|c|}{ MIC $(\mu \mathrm{g} / \mathrm{mIL}) \quad(n=104)$} \\
\hline & 125 & 62.5 & 31.25 & 15.62 & 7.8 & 3.9 & 1.95 & 0.97 & 0.48 & MIC Range & MIC $_{-50}$ & MIC $_{-90}$ \\
\hline Gentamicin & 7.7 & 13.5 & 3.8 & 2.9 & 15.4 & 11.5 & 10.5 & 13.5 & 21.2 & $0.48-125$ & 3.9 & 62.5 \\
\hline Oxytetracycline & 19.2 & 24 & 12.6 & 1.9 & 13.5 & 11.5 & 7.7 & 9.6 & - & $0.97-125$ & 31.25 & 62.5 \\
\hline Ceftriaxone & 11.5 & 13.5 & 6.7 & 2.9 & 11.5 & 9.6 & 9.6 & 23.1 & 11.5 & $0.48-125$ & 3.9 & 62.5 \\
\hline Enrofloxacin & 8.6 & 9.6 & 5.8 & 3.8 & 22.1 & 8.6 & 7.7 & 12.6 & 21.2 & $0.48-125$ & 3.9 & 31.25 \\
\hline Amoxicillin & 22.1 & 34.6 & 7.7 & 5.8 & 11.5 & 6.7 & 11.5 & - & - & $1.95-125$ & 62.5 & 125 \\
\hline $\begin{array}{l}\text { Pencillin G } \\
\text { Amoxicillin + }\end{array}$ & 21.2 & 44.2 & 2.9 & 13.5 & 8.6 & 4.8 & 2.9 & 1.9 & - & | 0.97-125 & 62.5 & 125 \\
\hline Sulbactam & - & 4.8 & 32.7 & 2.9 & 8.6 & 14.4 & 14.4 & 18.3 & 3.8 & $0.48-62.5$ & 3.9 & 31.25 \\
\hline Oxacillin & 14.4 & 24 & 14.4 & 14.4 & 11.5 & | 17.3 & 1.9 & - & - & $1.95-125$ & 31.25 & 125 \\
\hline
\end{tabular}

Numbers indicate percentage of isolates. Numbers in bold indicate percentage of isolates with MIC values greater than the highest concentration in the dilution range and vertical line indicate clinical breakpoints, with the value to the right of the line being susceptible and those to the left being resistant. MIC $_{50}$ and MIC $_{90}$ values are concentrations at which $\geq 50 \%$ and $\geq 90 \%$ of isolates are inhibited respectively.

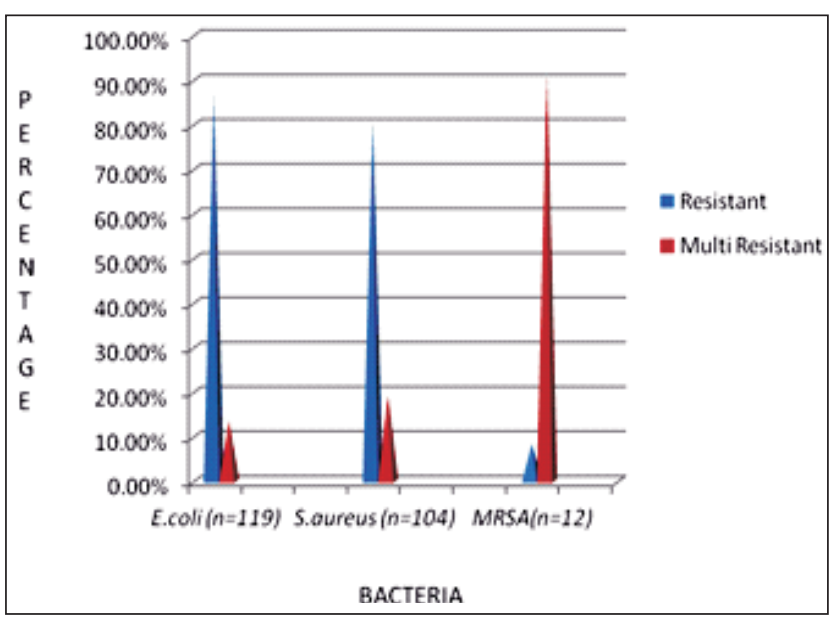

Figure-1. Incidence of resistance mastitis in cows

bials and few $E$. coli isolates (13.45\%) were found to be multi-drug resistant (Figure-1) i.e resistance to 3 or more of antimicrobials.

S. aureus isolates were most sensitive to enrofloxacin $(79.8 \%)$ followed by gentamicin $(71.2 \%)$, amoxicillin and sulbactam (69.2\%) and ceftriaxone $(69.2 \% \mathrm{t})$. The isolates showed highest resistance to penicillin $(63.5 \%)$ followed by amoxicillin $(61.5 \%)$, oxytetracycline (49\%) and methicillin (52.9\%). Most of the $S$. aureus isolates $(80.77 \%)$ were found to be resistant i.e resistance to 1 or 2 antimicrobials and few $S$. aureus isolates $(19.23 \%)$ were found to be multidrug resistant (Figure-1) i.e resistance to 3 or more antimicrobials.
MRSA showed maximum sensitivity to enrofloxacin (75\%), amoxicillin and sulbactam $(75 \%)$ followed by gentamicin $(66.7 \%)$ and ceftriaxone $(58.3 \%)$. The isolates showed highest resistance to methicillin (100\%), amoxicillin (91.7\%), followed by penicillin (83.3\%) and oxytetracycline (41.7\%). Few MRSA isolates $(8.33 \%)$ were found to be resistant i.e resistance to 1 or 2 antimicrobials and most of the MRSA isolates (91.67\%) were found to be multi-drug resistant (Figure-1) i.e resistance to 3 or more of antimicrobials.

MIC: Minimum inhibitory concentration of common antibiotics against E. coli, S.aureus and MRSA are presented in Table-2, 3 and 4.

The breakpoints for Enrofloxacin, oxytetracycline, amoxicillin, oxytetracycline, ceftriaxone, penicillin $\mathrm{G}$, oxacillin, amoxicillin + sulbactam and gentamicin were $\geq 2,8,16,32,16,16,16$ and $\geq 16 \mu \mathrm{g} / \mathrm{mL}$ for $E$. coli respectively.

Based on the breakpoints, the results indicated that $E$. coli was sensitive to gentamicin $(56.3 \%)$, enrofloxcain $(56.3 \%)$, amoxicillin + sulbactam $(50.4 \%)$, ceftriaxone (86.6\%) and resistant to amoxicillin (53\%), oxytetracycline $(58 \%)$, penicillin $G(60.5 \%)$ and oxacillin $(56.3 \%)$.

The breakpoints for penicillin, amoxicillin, oxacillin, ceftriaxone, enrofloxacin, amoxicillin + sulbactam, gentamicin, oxytetracycline, oxacillin and ceftriaxone were $\geq 0.25, \geq 0.5, \geq 4, \geq 4, \geq 4, \geq 8, \geq 16$ and $\geq 16$ 
Table-4: MIC of drugs against MRSA $(n=12)$ isolated from udder of cows

\begin{tabular}{|c|c|c|c|c|c|c|c|c|c|c|c|c|}
\hline \multirow[t]{2}{*}{ Antibiotics } & \multicolumn{12}{|c|}{ MIC $(\mu \mathrm{g} / \mathrm{mIL}) \quad(n=12)$} \\
\hline & 125 & 62.5 & 31.25 & 15.62 & 7.8 & 3.9 & 1.95 & 0.97 & 0.48 & MIC Range & MIC $_{-50}$ & MIC $_{-90}$ \\
\hline Gentamicin & 33.3 & 25 & 16.7 & - & 8.3 & 8.3 & 8.3 & - & - & $1.95-125$ & 31.25 & 125 \\
\hline Oxytetracycline & 25 & 41.7 & 16.7 & - & - & 8.3 & 8.3 & - & - & $1.95-125$ & 62.25 & 125 \\
\hline Ceftriaxone & 16.7 & 33.3 & 33.3 & - & 8.3 & 8.3 & - & - & - & $3.9-125$ & 62.25 & 62.5 \\
\hline Enrofloxacin & - & 16.7 & 16.7 & 16.7 & 8.3 & 25 & 8.3 & - & 8.3 & $0.48-62.5$ & 15.62 & 62.5 \\
\hline Amoxicillin & 33.3 & 50 & 16.7 & - & - & - & - & - & - & $31.25-125$ & 62.5 & 125 \\
\hline $\begin{array}{l}\text { Pencillin G } \\
\text { Amoxicillin + }\end{array}$ & 33.3 & 41.7 & 25 & - & - & - & - & - & - & $31.25-125$ & 62.5 & 125 \\
\hline Sulbactam & 8.3 & 25 & 16.7 & - & - & | 33.3 & 16.7 & - & - & $1.95-125$ & 31.25 & 62.5 \\
\hline Oxacillin & 66.7 & 33.3 & - & - & - & - & $1-$ & - & - & $62.5-125$ & 125 & 125 \\
\hline
\end{tabular}

Numbers indicate percentage of isolates. Numbers in bold indicate percentage of isolates with MIC values greater than the highest concentration in the dilution range and vertical line indicate clinical breakpoints, with the value to the right of the line being susceptible and those to the left being resistant. $\mathrm{MIC}_{50}$ and $\mathrm{MIC}_{90}$ values are concentrations at which $\geq 50 \%$ and $\geq 90 \%$ of isolates are inhibited respectively.

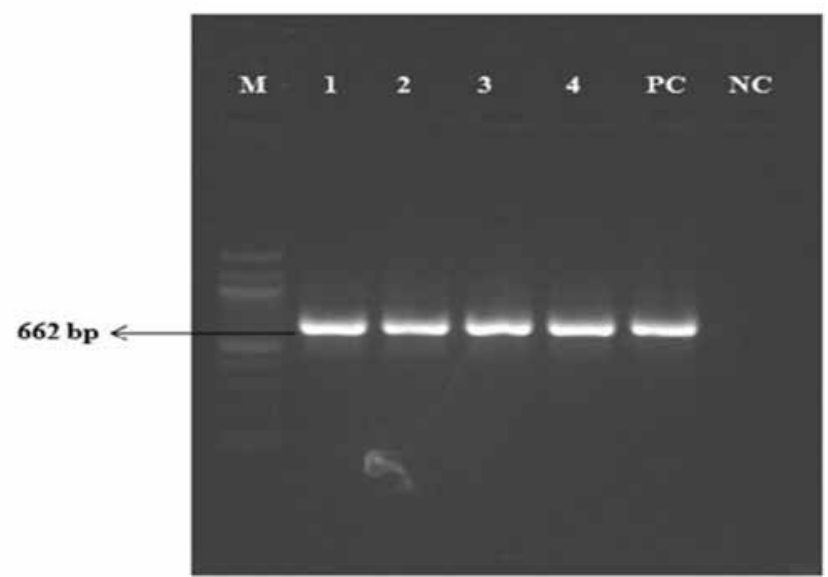

Figure-2: Amplification of 16s-23s rRNA gene of E. coli. M: 100bp ladder, 1-4: E. coli test samples, PC: Positive control, NC: Negative control.

$\mu \mathrm{g} / \mathrm{ml}$ for $S$. aureus respectively.

Based on the breakpoints, the results indicated that $S$. aureus was sensitive to gentamicin $(56.7 \%)$, enrofloxcain $(50.1 \%)$, amoxicillin $+\operatorname{sulbactam}(50.9 \%)$, ceftriaxone $(53.8 \%)$ and resistant to amoxicillin $(100 \%)$, oxytetracycline (55.8\%), penicillin G $(100 \%)$ and oxacillin $(80.8 \%)$.

The breakpoints for penicillin, amoxicillin, enrofloxacin, oxacillin, amoxicillin + sulbactam, ceftriaxone, gentamicin and oxytetracycline were $0.12,0.25, \geq 0.5$, $2,4,4,8$ and $8 \mu \mathrm{g} / \mathrm{ml}$ for MRSA respectively.

Based on the breakpoints, the results indicate that MRSA was sensitive to gentamicin (24.9\%), enrofloxcain $(8.3 \%)$, amoxicillin + sulbactam $(50 \%)$, ceftriaxone $(8.3 \%)$ and resistant to amoxicillin $(100 \%)$, oxytetracycline $(73.4 \%)$, penicillin $\mathrm{G}(100 \%)$ and oxacillin $(100 \%)$.

\section{Confirmation of the mastitis bacteria by targeting} specific genes for different strains: Out of $235 \mathrm{milk}$ samples, specific target gene of 16s-23s rRNA (E. coli) of 662 bp (Figure-2) could be amplified from 119 isolates with a percentage of positivity as 50.64 (119/235), the $229 \mathrm{bp}$ of 16s-23s rRNA (S. aureus) could be amplified from 104 isolates (Figure-3) with a percentage of positivity as $44.25(104 / 235)$. Screening for the specific target gene for both mecA (MRSA) of $513 \mathrm{bp}$ (Figure-4) and blaZ (MRSA) of $639 \mathrm{bp}$ (Figure-

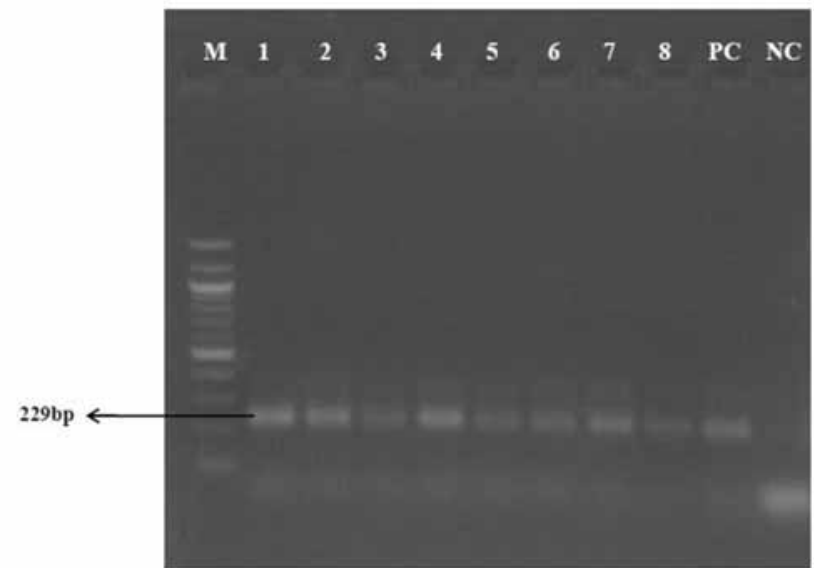

Figure-3: Amplification of 16s-23s rRNA gene of $S$. aureus. M: 100bp ladder, 1-8: S. aureus test samples, PC: Positive control, NC: Negative control.

5 ) resulted in positivity in 12 samples with a percentage of positivity as $10.34(12 / 116)$ among the $S$. aureus isolate.

\section{Discussion}

In the present study, no resistance were observed for the Streptococcus spp. and Bacillus spp. Antimicrobial resistance has been reported to be most common among the Staphylococcal mastitis isolates with a much lower proportion of Streptococcal isolates exhibiting resistance [14]. The antibiotic usage has directly contributed to an increased prevalence of resistant $E$. coli mastitis [15]. All antimicrobial use in the herd may affect the resistance of $E$. coli isolates by increasing the presence of these antimicrobial agents in the cow's environment. The incidence of resistant $S$. aureus mastitis was higher which might be due to indiscriminate use of antibiotics and intramammary preparations containing combinations and broadspectrum antibiotics [16].

MRSA strains have been observed to be multidrug resistant, such as aminoglycosides, macrolides, lincosamides, streptogramins, tetracyclines, etc., which are often used in the treatment of mastitis [17]. In the present study, multidrug resistance to methicillin, amoxicillin, penicillin and oxytetracycline was commonly observed in MRSA mastitis. MRSA strains 


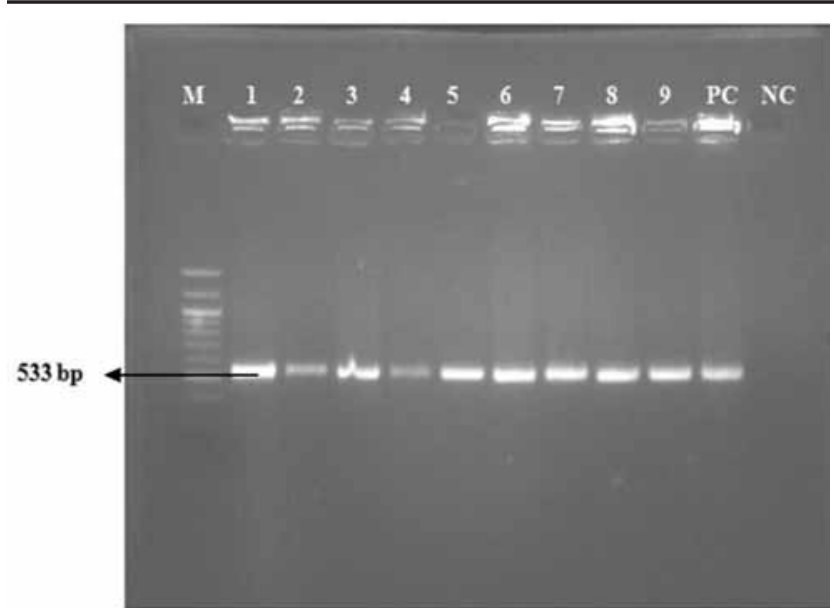

Figure-4: Amplification of mecA gene of MRSA. M: 100bp ladder, 1-9: MRSA test samples, PC: Positive control, NC: Negative control.

were multi-drug resistant which might be due to production of betalactamase and PBP2a (penicillin binding protein) [17]. A previous study indicates the prevalence of 13 percent of $S$. aureus MRSA isolates from cows with mastitis in a herd located in northwest India [2]. High incidence in the present study might be due to indiscriminate use of antibiotics and consequent transfer of plasmid mediated antibiotic resistance and concurred with above authors. Indiscriminate use of antibiotics and intramammary preparations used by the owner without the prescription of the veterinarian is also attributed to be one of the reasons for increasing incidence of these strains.

Gram negative pathogens were more sensitive to enrofloxacin and gentamicin and less sensitive to ampicillin and penicillin [18]. In the present study, incidence of resistant $E$. coli mastitis was higher which could be due to the wide antibiotic usage [15].

The data for $\mathrm{MIC}_{50}$, clinical breakpoints of amoxicillin, penicillin $\mathrm{G}$, oxacillin and amoxicillin + sulbactum against $E$. coli were not available. However, the clinical break point of ampicillin and amoxicillinclavulanate were taken for the present study.

The breakpoints for gentamicin and enrofloxacin were $\geq 16$ and $\geq 2 \mu \mathrm{g} / \mathrm{ml}$ for E.coli respectively [19]. The breakpoints for amoxicillin, oxytetracycline, ceftriaxone, penicillin $\mathrm{G}$, oxacillin and amoxicillinclavulanate were $16,8,32,16,16$ and $16 \mu \mathrm{g} / \mathrm{mL}$ for $E$. coli respectively [20].

Based on the MIC break point for pencillin G, oxytetracycline, amoxicillin and oxacillin in $E$. coli mastitis, they were considered as resistance and could be attributed to the indiscriminate use of these drugs. It highlights the need for systematic study of resistance pattern before initiating antibiotic therapy.

The data for $\mathrm{MIC}_{50}$, clinical breakpoints of amoxicillin + sulbactum against $S$. aureus were not available. However, the clinical break point for amoxicillin-clavulanate were included in the study.

The breakpoints for amoxicillin, amoxicillin + clavulanate, enrofloxacin, gentamicin, oxytetracycline, penicillin and oxacillin were $\geq 0.5, \geq 8, \geq 4, \geq 16, \geq 16$,

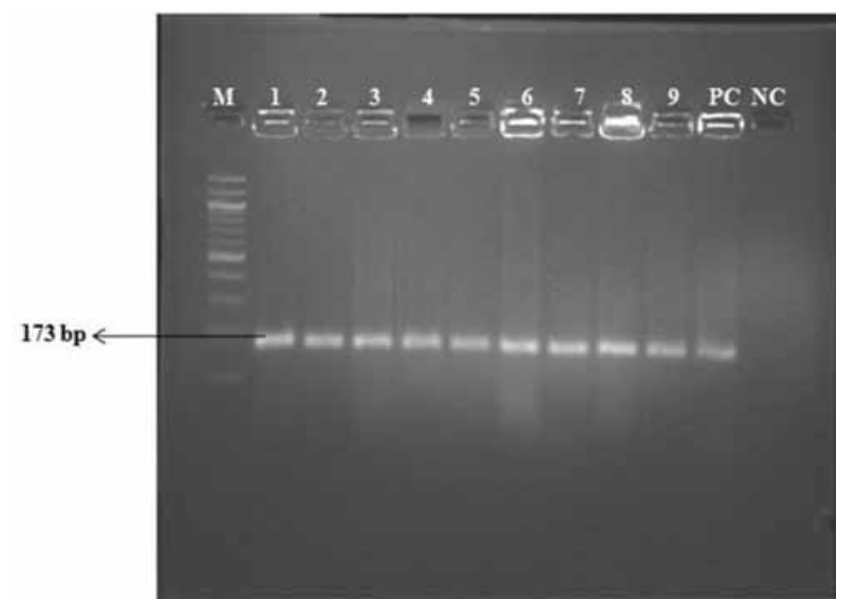

Figure-5: Amplification of blaZ gene of MRSA. M: 100bp ladder, 1-9: MRSA test samples, PC: Positive control, NC: Negative control.

$\geq 0.25$ and $\geq 4 \mu \mathrm{g} / \mathrm{ml}$ for $S$. aureus respectively [19]. The breakpoints for ceftriaxone were $\geq 4 \mu \mathrm{g} / \mathrm{mL}$ for $S$. aureus [20]. The high resistance of pencillin G, oxytetracycline, amoxicillin and oxacillin in S.aureus mastitis in the present study could be attributed to the indiscriminate use of these drugs and intramammary preparations used by the owner without the prescription of the veterinarian.

The breakpoints for gentamicin, oxytetracycline, ceftriaxone, enrofloxcain, amoxicillin, penicillin $\mathrm{G}$, amoxicillin + sulbactam and oxacillin were $8,8,4, \geq$ $0.5,0.25,0.12,4$, and $2 \mu \mathrm{g} / \mathrm{mL}$ for MRSA respectively [9]. The high resistance of pencillin $\mathrm{G}$, oxytetracycline, amoxicillin and oxacillin in MRSA mastitis in the present study could be attributed to the indiscriminate use of these drugs and intramammary preparations used by the owner without the prescription of the veterinarian.

Based on the antibiotic susceptibility test and MIC break points, E. coli, S. aureus and MRSA showed maximum sensitivity to enrofloxacin, amoxicillin and sulbactam followed by gentamicin and ceftriaxone.

These susceptible antibiotic drugs will be used as the effective drugs against E. coli, S. aureus and MRSA resistant isolates. The present study demonstrated that the resistant strains may be transferred to milk from infected udders, poor farm practices and due to poor handling during milking, transmission to the milk utensils, which could be the reason for infection in human beings.

The present study has demonstrated the existence of alarming levels of resistance of E. coli, S. aureus and MRSA to commonly used antimicrobial agents in the study farms and the results are in accordance with reports from earlier studies in other countries. Edward et al., [21] suggesting a possible development of resistance from prolonged and indiscriminate usage of some antimicrobials. It is therefore, very important to implement a systemic application of an in vitro antibiotic susceptibility test prior to the use of antibiotics in both treatment and prevention of intra-mammary infections. 
Only a few studies in India have been carried out to assess MRSA status among mastitis infections [2]. The presence of MRSA signifies alarming levels of resistance. Further it highlights the need for preventing the indiscriminate use of antibiotics. Avoiding routine antimicrobial use in food animals and to decrease selection pressure against anti-microbials, might decrease the prevalence of MRSA among cows.

\section{Conclusion}

AMR prevalence was more common in bovine mastitis pathogens namely $E$. coli and $S$. aureus. Overall, antimicrobial resistance to penicillin, amoxicillin, oxytetracycline and methicillin was found in clinical mastitis cases. Multidrug resistance was more commonly observed in MRSA isolates than in $E$. coli and $S$. aureus isolates. 12 out of $116 S$. aureus isolates screened was positive for MRSA (Prevalence of $10.34 \%$ ). This study result suggests that a high risk for transmission of AMR bacteria from milk or milk products to human populations.

\section{Authors' contributions}

DC planned, designed and carried out research work under his $\mathrm{Ph} . \mathrm{D}$ thesis programme in collaboration with guide APN and advisory members PST, KK and SV. PV and KGT assisted in the designing and performance of PCR and SR assisted in the designing of MIC. DC and KGT revised the manuscript. All authors read and approved the final manuscript.

\section{Acknowledgements}

The current research has been carried out at the Department of Veterinary Clinical Medicine, Ethics and Jurisprudence, Madras Veterinary College, Tamil Nadu Veterinary and Animal Sciences University (TANUVAS), Chennai, India. The study is a part of the post graduate research work, "Evaluation of antibiotic resistant mastitis in dairy cows" and submitted by the first author to TANUVAS, Chennai. The support for part of the study is by DST-TDT division, Government of India, New Delhi for developing a somatic cell assay.

\section{Competing interests}

The authors declare that they have no competing interests.

\section{References}

1. Barkema, H.W., Schukken, Y.H. and Zadoks, R.N. (2006) Invited review: The role of cow, pathogen, and treatment regimen in the therapeutic success of bovine Staphylococcus aureus mastitis. J. Dairy Sci., 89: 1877-1895.

2. Kumar, R., Yadav, B.R. and Singh, R.S. (2011) Antibiotic resistance and pathogenicity factors in Staphylococcus aureus isolated from mastitic Sahiwal cattle. J. Bio. Sci., 36: 175-188.

3. Olsen, J.E., Christensen, H. and Aarestrup, F.M. (2006) Diversity and evolution of blaZ from Staphylococcus aureus and Coagulase negative Staphylococci. J. Antimicrob. Chemother, 57, 450-460.

4. Turutoglu, H., Ercelik, S. and Ozturk, D. (2006) Antibiotic resistance of Staphylococcus aureus and Coagulasenegative Staphylococci isolated from bovine mastitis. Bull. Vet. Inst. Pulawy., 50: 41-45.
5. Vanderhaeghen, W., Cerpentier, T., Adriaensen, C., Vicca, J., Hermans, K. and Butaye, P. (2010) Methicillin-resistant Staphylococcus aureus (MRSA) ST398. associated with clinical and subclinical mastitis in Belgian cows. Vet. Microbiol., 144: 166-171.

6. Juhász-Kaszanyitzky, E., Janosi, S., Somogyi, P., Dan, A., Bloois, L.G. and Wagenaar, J.A. (2007) MRSA transmission between cows and humans. Emer. Infect. Dis., 13, 630-632.

7. Piddock, L.J. (1996) Does the use of antimicrobial agents in veterinary medicine and animal husbandry select antibioticresistant bacteria that infect man and compromise antimicrobial chemotherapy? J. Antimicrob. Chemother., 38: 1-3.

8. CLSI. (2008) Performance standards for antimicrobial disk and dilution susceptibility tests for bacteria isolated from Animals. Pages 65-72. In: Approved standard M31-A3(3 ${ }^{\text {rd }}$ edn). Clinical and Laboratory Standards Institute, Wayne, PA.

9. Sarker, S.D., Nahar, L. and Kumarasamy,Y. (2007) Microtitre plate-based antibacterial assay incorporating resazurin as an indicator of cell growth, and its application in the in vitro antibacterial screening of phytochemicals. Sci. Dir. Meth., 42:321-324.

10. Pradhan, P., Gopinath, S.M., Reddy, G.R., Dechamma, H.J. and Suryanarayana,V.V.S. (2011) Detection of major pathogens in bovine subclinical mastitis by multiplex PCR directly from milk samples in presence of an internal control. Ind. J. Fund and App. Life Sci., 1(4): 209- 218.

11. Riffon, R., Sayasith,K., Khalil,H., Dubreuil, P., Drolet, M. and Lagace, J. (2001) Development of a rapid and sensitive test for identification of major pathogens in bovine mastitis by PCR. J. Clin. Microbiol., 39(7): 2584-2589.

12. Lee, J.H. (2003) Methicillin(oxacillin)-resistant Staphylococcus aureus strains isolated from major food animals and their potential transmission to humans. Appl. Environ. Microbiol., 69: 6489-6494.

13. Martineau, F., Picard, F.J., Lansac, N., Ménard, C., Roy, P.H., Ouellette, M. and Bergeron,M.G. (2000) Correlation between the resistance genotype determined by multiplex PCR assays and the antibiotic susceptibility patterns of Staphylococcus aureus and Staphylococcus epidermidis. Antimicrob. Agents Chemother., 44: 231-238.

14. Call, D.R., Davis, M.A. and A.A. Sawant, A.A. (2008). Antimicrobial resistance in beef and dairy production. Anim. Health Res. Rev., 9: 159-167.

15. DANMAP. (2001) Use of antimicrobial agents and occurrence of antimicrobial resistance in bacteria from food animals, foods and humans in Denmark. The Danish Integrated Antimicrobial Resistance Monitoring and Research Programme, Copenhagen, Denmark.

16. Pitkala, A., Salmikivi,L., Bredbacka, P., Myllyniemi, A.L. and Koskinen,M.T. (2004) Comparison of tests for detection of beta-lactamase-producing Staphylococci. J. Clin. Microbiol., 45: 2031-2033.

17. Kumar, R., Yadav, B.R. and Singh, R.S. (2010) Genetic Determinants of antibiotic resistance in Staphylococcus aureus isolates from milk of mastitic crossbred cattle. Curr. Microbiol., 60: 379-386.

18. Karthikeyan, A. (2003) Clinicopathological and ultrasonographic studies in bovine mastitis. M.V.Sc., Thesis submitted to Tamilnadu Veterinary and Animal Sciences University, Chennai, India.

19. Kumar, A., Singh, U., Kumar, S., Sengar,G., Singh, Singh, R., Sharma, A. and Rajib Deb, E. (2013) Phylogenetic analysis of Escherichia coli isolated from mastitis milk samples. Adv. Anim. Vet. Sci., 1(1S): 9-10.

20. Febler, A., Scott, C., Kadlec, K., Ehricht, R., Monecke, S. and Schwarz, S. (2010) Characterization of methicillinresistant Staphylococcus aureus ST398 from cases of bovine mastitis. J. Antimicrob. Chemother., 65(4): 619-25.

21. Edward, M., Anna, K., Michal, K., Henryka, L. and Krystyna, K. (2002) Antimicrobial susceptibility of Staphylococci isolated from mastitic cows. Bull. Vet. Inst. Pulawy., p 289-294. 